\title{
2
}

\section{The Anatomy of the Hippocampus}

\author{
Pradip Chauhan $^{1} \bullet$ Kinjal Jethwa ${ }^{2} \bullet$ Ashish Rathawa $^{3} \bullet$ Girish Chauhan ${ }^{4} \bullet$ \\ Simmi Mehra ${ }^{1}$ \\ ${ }^{1}$ Department of Anatomy, All India Institute of Medical Sciences, Rajkot, Gujarat, India; \\ ${ }^{2}$ Department of Anatomy, SKBS Medical College and Research Institute, Sumandeep \\ Vidhyapeeth, Baroda, Gujarat, India; ${ }^{3}$ Department of Anatomy, GMERS Medical College, \\ Junagadh, Gujarat, India; ${ }^{4}$ Department of Oral Pathology, Government Dental College, \\ Jamanagar, Gujarat, India.
}

Author for correspondence: Pradip Chauhan, Department of Anatomy, All India Institute of Medical Sciences, Rajkot, Gujarat, India. Email: prajjawalitresearch@gmail.com

Cite this chapter as: Chauhan P, Jethwa K, Rathawa A, Chauhan G, Mehra S.

The Anatomy of the Hippocampus. In: Pluta R, editor. Cerebral Ischemia. Brisbane (AU):

Exon Publications; 2021. Online first Sep 17.

Doi: https://doi.org/10.36255/exonpublications.cerebralischemia.2021.hippocampus

\begin{abstract}
The hippocampal formation is responsible for memory processing, learning, spatial navigation, and emotions. It includes the indusium griseum, longitudinal striae, gyrus fasciolaris, hippocampus proper (cornu ammonis, dentate gyrus, and subiculum) and part of the uncus. The hippocampus has the archipallial cortex and is formed by the infoldings of the dentate gyrus, cornu ammonis and subiculum. The dentate gyrus is a narrow crenated strip of grey matter. The dentate gyrus consists of three layers, from the outside in: the molecular layer, granular layer, and polymorphic layer. The granular neurons receive input from the parahippocampal gyrus (entorhinal cortex) via the perforant pathway. The granular neurons send mossy fibers to the apical dendrites of pyramidal cells present in the cornu ammonis. The axons of hippocampal pyramidal cells form a sheet of white fibers known as the alveus which continues as fimbria and fornix. The fornix projects into the septal area. From the septal area few fibers synapse into the cingulate gyrus which returns to the hippocampus. The neuronal intrinsic circuit, known as the Papez circuit of the hippocampus, plays a crucial role in the memory processing.
\end{abstract}

Keywords: fimbria; fornix; hippocampus; Papez circuit; perforant pathway

In: Cerebral Ischemia. Pluta R (Editor). Exon Publications, Brisbane, Australia. ISBN: 978-0-6450017-9-2; Doi: https://doi.org/10.36255/exonpublications. cerebralischemia.2021

Copyright: The Authors.

License: This open access article is licenced under Creative Commons Attribution-NonCommercial 4.0 International (CC BY-NC 4.0) https://creativecommons.org/licenses/by-nc/4.0/ 


\section{INTRODUCTION}

The hippocampus is part of the limbic lobe buried in the medial part of the temporal lobe and involved in memory processing, learning, spatial navigation, and emotions (1). The hippocampal formation (formatio hippocampi) comprises (Figure 1) indusium griseum, longitudinal striae, gyrus fasciolaris, hippocampus proper (cornu ammonis, dentate gyrus and subiculum) and part of the uncus (1-3). The hippocampal formation has archipallial cortex and develops along the inferomedial surface of cerebral hemisphere; during development, it follows the outer border of the C-shaped choroidal fissure (4). The hippocampus converts short-term memory into long-term memory, solves spatial memory, and recollects the past experiences of places. It also plays a pivotal role in emotions and behavior of a person.
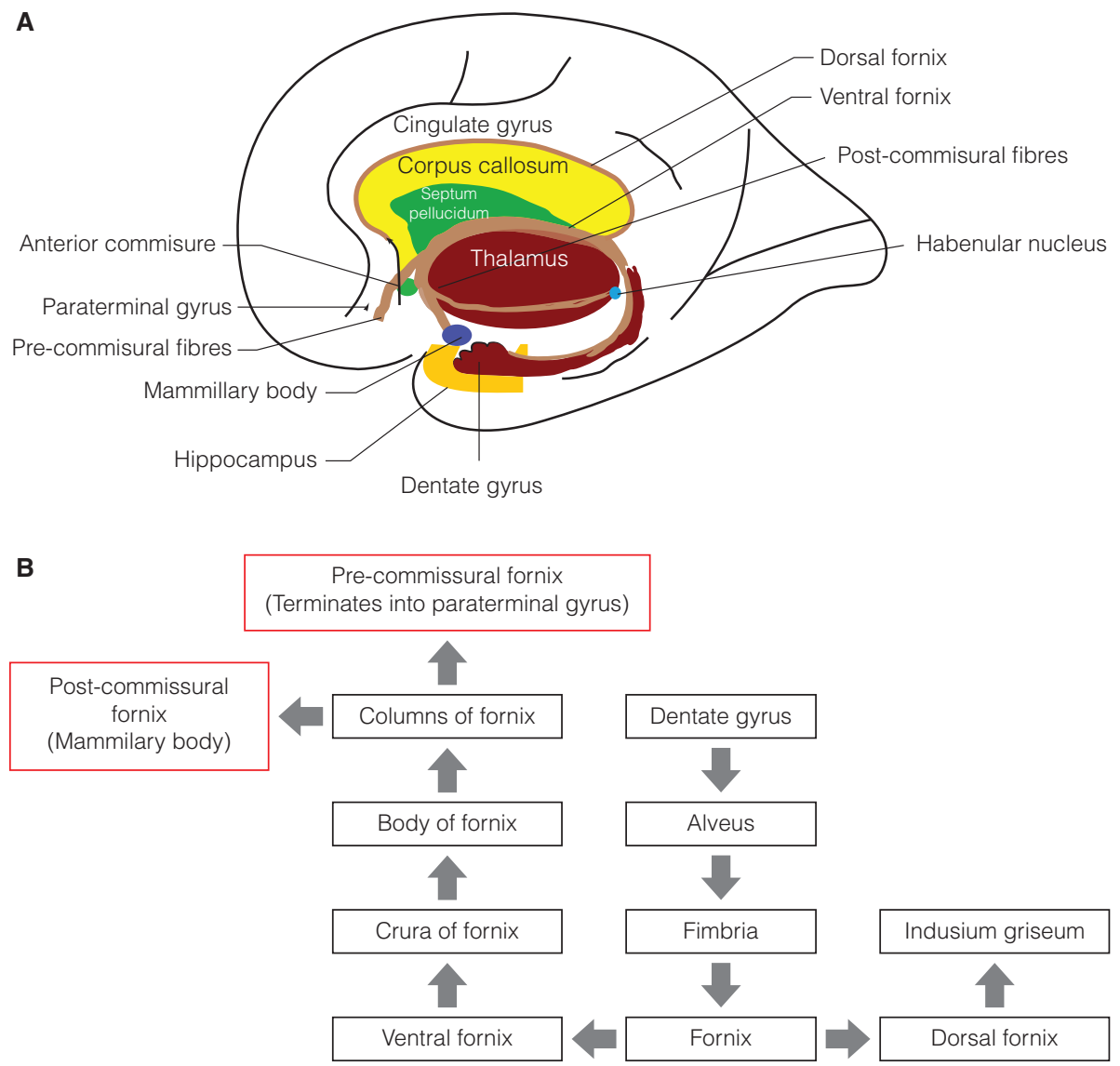

Figure 1. Hippocampal formation and connection of fornix. A, Schematic diagram of medial surface of the cerebral hemisphere showing dentate gyrus, cingulate gyrus, fornix, mammillary body and paraterminal gyrus. B, Formation, course, and termination of fornix. 


\section{INDUSIUM GRISEUM AND LONGITUDINAL STRIAE}

Indusium griseum and longitudinal striae are vestigial parts of the hippocampal formation (5). Indusium griseum is a thin sheet of grey matter above the corpus callosum and traversed by pairs of medial and lateral longitudinal striae on each side of the midline $(5,6)$. Anteriorly, the indusium griseum continues around the genu and rostrum of the corpus callosum with the paraterminal gyrus (5-7). The indusium griseum continues with the gyrus fasciolaris posteriorly around the splenium of the corpus callosum (Figure 1) and then with the dentate gyrus (5-7). The indusium griseum is continuous laterally with the cingulated gyrus through the callosal sulcus (7).

\section{DENTATE GYRUS}

The dentate gyrus (Figure 2) is a narrow crenated strip of grey matter and continuation of gyrus fasciolaris along the upper surface of the parahippocampal gyrus (8). The dentate gyrus contains archtitectorial fascia dentata that consists of three layers (Figure 2). Superficial to deep, the layers are: molecular layer, intermediate granular layer, and multiform layer (8-10). The multiform layer continues with the CA4 region (hilus) of the cornu ammonis. The hilus and fascia dentate collectively form the dentate gyrus $(8,9)$. The dentate gyrus is continuous as a tail of the dentate gyrus (band of Giacomini) backwards and medially across the uncus $(8,9)$. The band of Giacomini divides the uncus into two parts: the uncinate gyrus anterior to the band of Giacomini and intralimbic gyrus posterior to the band of Giacomini (8-11). The dentate gyrus is separated from the parahippocampal gyrus by the hippocampal sulcus (8-11). Fimbria of fornix that covers the dentate gyrus is separated from the dentate gyrus by the fimbrio-dentate sulcus (8-11).

\section{HIPPOCAMPUS}

Hippocampus is an elongated convex structure deep in the medial temporal lobe and presents an elevation (Figures 1 and 2) along the floor of the inferior horn of the lateral ventricle $(1,2,4)$. The hippocampus has the archipallial cortex and is formed by the infoldings of the dentate gyrus, cornu ammonis and subiculum $(1,4,9-11)$. The subiculum is continuous with the six-layered neocortex (parahippocampal gyrus). During development, cornu ammonis and dentate gyrus are folded into the inferior horn of the lateral ventricle at the hippocampal sulcus (8-10); the process brings the outer molecular layers of the dentate gyrus and subiculum close to each other (12).

The shape of the hippocampus in gross dissection looks like a seahorse (genus Hippocampus) on the basis of which the structure is termed as 'Hippocampus'. The hippocampus is also known as 'Ammon's horn' because the C-shaped coronal section of the hippocampus resembles ram's horn; the term 'Ammon's horn is derived from the Egyptian deity with ram's head (2). The hippocampus is also 
A

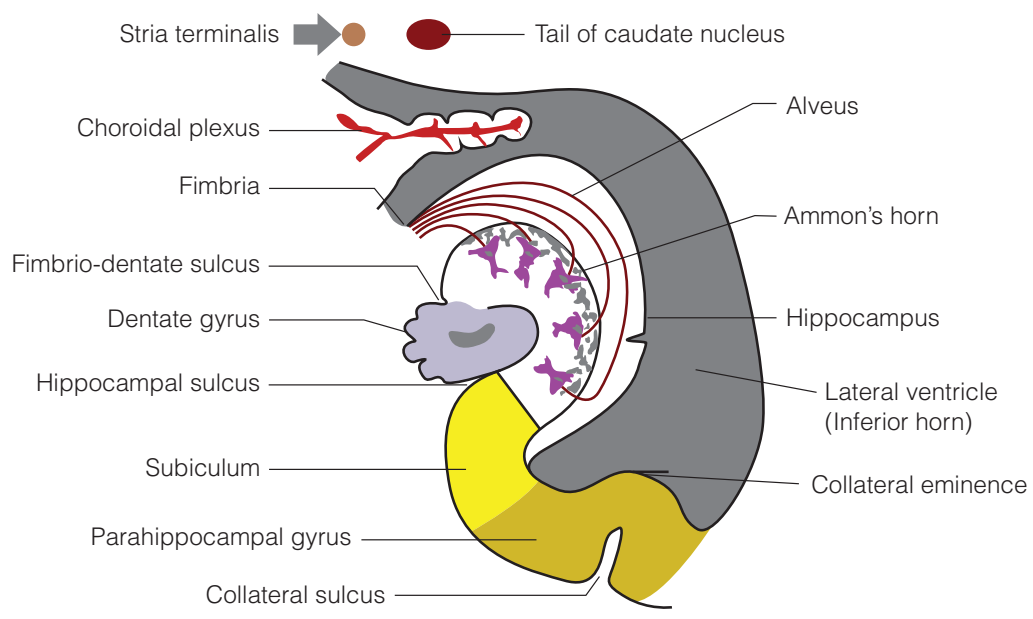

B

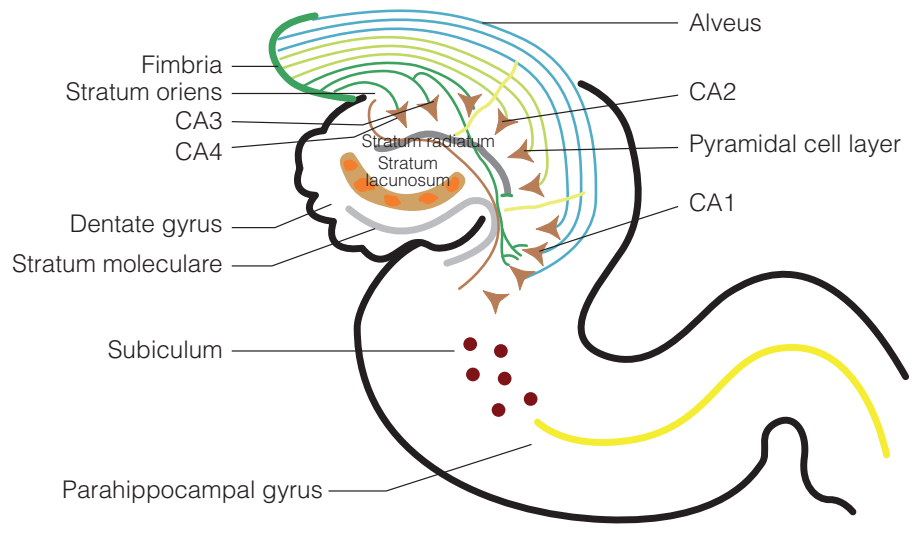

Figure 2. The hippocampus, dentate gyrus, subiculum, and entorhinal cortex. A, Coronal section through the hippocampus and dentate Gyrus; B, Schematic diagram to show the histological layers of the dentate gyrus and cornu ammonis.

known as 'pes hippocampi' because its anterior bulbous extremity is marked by a number of grooves and the feature resembles a paw of an animal $(4,11)$. The alveus, a thin sheet of white matter, covers the ventricular surface of the hippocampus $(10,11)$. The axons of hippocampal pyramidal cells form the alveus; the fibers of the alveus converge at the medial margin of the hippocampus to form fimbria hippocampi $(4,10,11)$. The fimbria hippocampi proceed posteriorly covering the dentate gyrus and reach to the splenium of the corpus callosum; thereafter, continues with the fornix $(2,4,10,11)$. The fimbrio-dentate sulcus separates the fimbria and the dentate gyrus. The fimbria continues as fornix around the thalamus separated by choroidal fissure containing choroidal plexus (9-11). 


\section{MICROSCOPIC STRUCTURE}

The dentate gyrus is the input channel of the hippocampal formation. Histologically, the dentate gyrus consists of three layers (Figure 2), from the outside in: the molecular layer, granular layer, and polymorphic layer $(13,14)$. The dentate gyrus is semilunar in shape; convexity of which is directed towards the molecular layer while the concavity is directed towards the cornu ammonis $(2,4,10-14)$.

Dendrites of the granular neurons receive input from the para-hippocampal gyrus (entorhinal cortex) via perforant pathway (Figure 3) (1, 10, 14). The axons of the granular neurons synapse by mossy fibers with the apical dendrites of the pyramidal cells present in the cornu ammonis. The three layers of cornu ammonis (Figure 2) can be further subdivided into the following sublayers $(9,11,14-17)$ :

(i) Alveus: Efferent fibers from the axons of the pyramidal cells of cornu ammonis form the alveus while some axon collateral re-enters the hippocampus.

(ii) Stratum oriens: Contains a few inhibitory basket-cell interneurons; two types of basket cells are observed in the stratum oriens. Axons and dendrites of pyramidal cells, recurrent axon collateral, and commissural fibers traverse the stratum oriens.

(iii) Stratum pyramidalis: Forms the principal cellular component of cornu ammonis. 10-30 layers of pyramidal cells are present in the stratum pyramidalis; functionally, the pyramidal cells are excitatory. The pyramidal cells have an apex and a base; the base is directed toward the alveus while the apex is directed towards the outer molecular layer. Alveus and fimbria are formed by the axons arising from the center of the base of pyramidal cells. Dendrites arise from the base and apex of the pyramidal cells. The dendrites arising from the base ramify in the stratum oriens and the basal dendrites receive commissural fibers from identical areas of the contralateral hippocampus. Dendrites arising from the apex extend deeper to branch profusely and apical dendrites receive commissural fibers from non-identical areas of the contralateral hippocampus. Apical dendrites also receive afferents from the entorhinal areas and mossy fibers from the dentate gyrus. Recurrent collateral from the neighboring pyramidal cells synapses with the apical dendrites.

(iv) Stratum radiatum: Comprises apical dendrites of the pyramidal cells and some stellate cells.

(v) Stratum lacunosum-moleculare: Contains axons and interneurons. Inhibitory interneurons from the stratum lacunosum-moleculare project into the retrosplenial cortex.

\section{ORGANIZATION OF PYRAMIDAL CELLS IN HIPPOCAMPUS}

Cornu ammonis of the hippocampus can be subdivided into four regions (Figure 2, 3): CA1, CA2, CA3, and CA4 (13-17). The CA1 is the largest region and is delimited laterally by the presubiculum and medially by CA2. Most neurons (90\%) of CAl are pyramidal cells (glutamatergic projection neurons) and the 
A

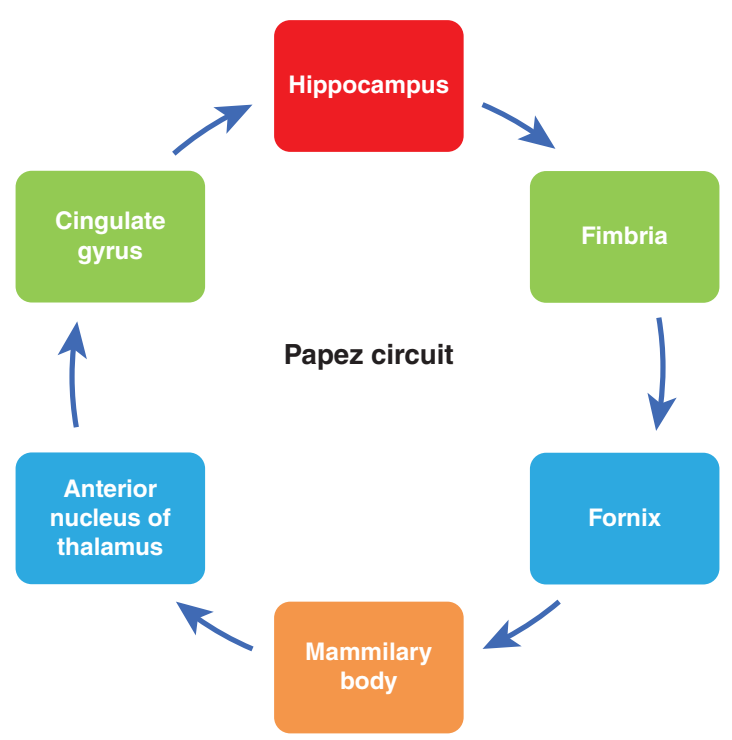

B

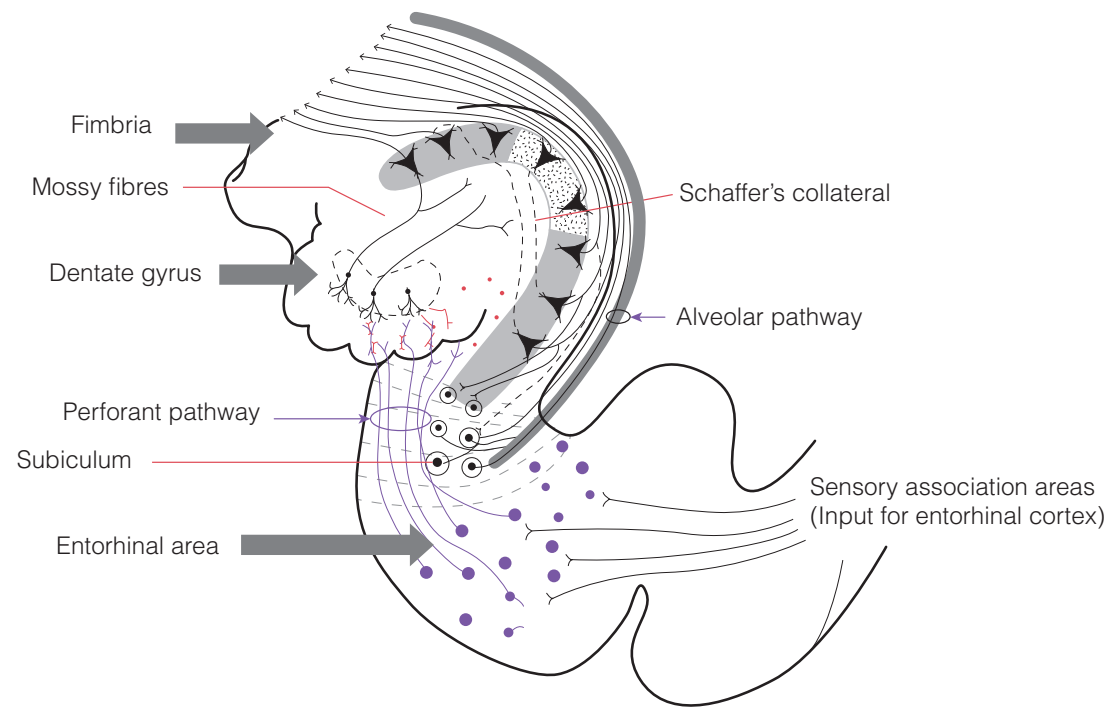

Figure 3. Intrinsic neuronal circuit of the hippocampal formation. A, Papez memory circuit. B, Perforant and alveolar pathway. 
C

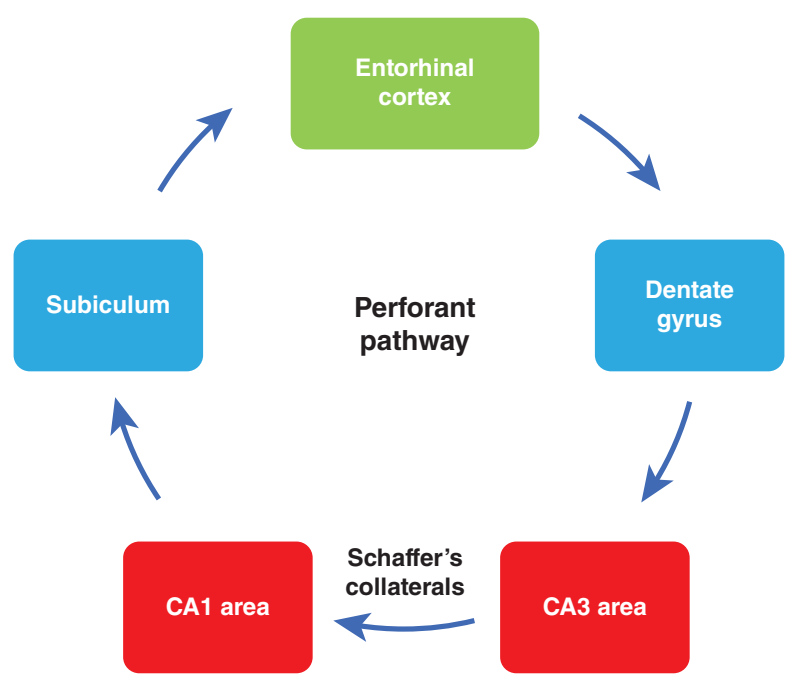

Figure 3. (Continued) Intrinsic neuronal circuit of the hippocampal formation. C, Perforant pathway.

rest $(10 \%)$ are interneurons. CA2 layer is present towards the dentate gyrus bounded laterally by the CA1 and medially by the CA3 layer. CA2 of the cornu ammonis receives input from the supramammilary region of the hypothalamus but lacks input mossy fires from the dentate gyrus. CA3 layer is directed towards the hilus of the dentate gyrus and is limited medially by the CA2 layer. The superficial most cells of the CA3 are described as CA4 by some authors. The apical dendrites of the CA3 layer receive mossy fibers from the granule cells of the dentate gyrus. Axons of the CA3 pyramidal cells contribute to the alveus, fimbria, and fornix. Some CA3 axons give collateral fibers known as the Schaffer's collaterals which synapse with the dendrites of CAl pyramidal cells. Axons from the CAl pyramidal cells are connected to the subiculum neurons. Axons from the subiculum neurons contribute to form the fibers of the fimbria and fornix via the alveolar pathway (Figure 3).

\section{CONNECTIONS OF HIPPOCAMPUS}

The hippocampus receives afferents from the following structures $(5,17-24)$ :

(i) Cingulate gyrus via cingulam.

(ii) The indusium griseum and septal nuclei through the fornix.

(iii) Contralateral hippocampus through the hippocampal commissure.

(iv) Outer part of the entorhinal area through the perforant pathway.

(v) Inner part of the entorhinal area and subiculum to the alveus through the alveolar pathway. 
Efferent fibers from the hippocampus (Figure 1) are connected to the following areas through the fornix (17-24):

(i) Gyrus fasciolaris, indusium griseum, cingulate gyrus, and septal nuclei through the fibers of the dorsal fornix.

(ii) Paraterminal gyrus, pre-optic, and anterior hypothalamic nuclei through the pre-commissural fornix.

(iii) Anterior nucleus of thalamus, hypothalamic nuclei, mammillary body through the post-commissural fibers.

(iv) Habenular nucleus via stria medullaris thalami.

\section{FORNIX}

Fornix forms efferent projection fibers of the hippocampus and also comprises commissural fibers connecting the hippocampus of both sides $(5,10,17-24)$.

\section{Formation of fornix}

Axons of pyramidal cells from the cornu ammonis with a major contribution from the subicular complex forms the alveus. Alveus continues as a fimbria which is separated from the dentate gyrus by the fimbrio-dentate sulcus. Continuation of the fimbria is called the fornix.

\section{Course of fornix}

Fornix is divided into dorsal fornix and ventral fornix at the splenium of the corpus callosum (Figure 1). The dorsal fornix runs along with the gyrus fasciolaris and indusium griseum surrounding the outer surface of the corpus callosum. The ventral fornix runs forward below the splenium of the corpus callosum and around the pulvinar of the thalamus. Ventral fornix forms a pair of crura that run forwards and converge to form the body of the fornix. Commissural fibers connect the medial margins of both crura through the commissure of the fornix (hippocampal commissures). Hippocampal commissures and the crura of the fornix are separated from the body of the corpus callosum by a space known as the ventricle of the fornix.

\section{Body of the fornix}

The body of the fornix is a triangular structure having two symmetrical halves and the apex of which is directed in front. Bilaminar septum pellucidum connects the superior surface of the body of the fornix to the body of the corpus callosum. The inferior surface is related to the ependymal roof of the third ventricle and the thalamus separated by tela choroidea (folding of pia mater containing the choroidal plexus) and a pair of internal cerebral veins. Laterally, choroidal fissure separates the body of the fornix from the thalamus. At the anterior interventricular foramen, the body of the fornix diverges into a pair of columns of the fornix (Figure 1). 
The anterior commissure separates the fibers of the column into pre-commissural fornix and post-commissural fornix. The pre-commissural fornix which mainly contains axon fibers from the CA3 pyramidal cells continues with the para-terminal gyrus. The post-commissural fornix forms the anterior boundary of the interventricular foramen and then turns downwards and backwards beneath the ependyma of the third ventricle to reach the mammillary body.

\section{HABENULAR NUCLEI}

The habenular nuclei are pairs of nuclei located in the habenular trigone. The habenular trigone is a depression on either side of the pineal stalk bounded by the stria medullaris (cranio-medially), superior colliculus (caudally), and pulvinar end of the thalamus (laterally) $(4,7,25-28)$. The habenular nuclei receive afferents via the stria medullaris thalami from the septal area (subcallosal area) and pre-optic nuclei (hypothalamus), via stria terminalis from the amygdaloid body and via fornix from the hippocampus $(1,4-8,25-28)$. Some stria medullaris thalami fibers pass through the dorsal lamina of the pineal stalk and connect two habenular nuclei. The commissural fibers connecting two habenular nuclei are known as habenular commissure. The habenular nuclei send efferent fibers to the interpeduncular nucleus through the fasciculus retroflexus of Meynert. The interpeduncular nucleus projects to the dorsal tegmental nucleus. Dorsal longitudinal fasciculus connects the dorsal tegmental nucleus to the autonomic and reticular nuclei of the brainstem $(1,5,25)$.

\section{MAMMILO-TEGMENTAL TRACT}

The mammilo-tegmental tract influences the brainstem and spinal cord for integrated motor response (25). The mammilo-tegmental tract is a collection of efferent fibers from the mammillary body to the midbrain tegmental nuclei. Efferent fibers from the tegmental nuclei reach the reticular nuclei as reticulo-bulbar and reticulo-spinal tract (26-28).

\section{MAMMILO-THALAMIC TRACT}

The mammilo-thalamic tract connects the mammillary body to the anterior nucleus of the thalamus (25-28). The efferent fibers from the anterior nucleus of thalamus reach the cingulate gyrus. From the cingulate gyrus, some fibers project back to the hippocampus.

\section{HIPPOCAMPAL FORMATION AND MEMORY}

One of the functions of the hippocampus is converting short-term memory into long-term memory. The long-term memory is maintained by a unidirectional 
progression of synaptic connections through the intrinsic hippocampal circuitry (Figure 3).

\section{Papez circuit}

Hippocampal circuitry postulated by Papez is called as 'Papez circuit' and is adapted as a memory circuit. The Papez circuit is believed to be responsible for emotional integration and for recent memory trace (1, 4, 7-10, 25-28). The Papez circuit includes the hippocampus, fimbria, fornix, mammillary body, anterior nucleus of the thalamus and cingulate gyrus (Figure 3A). The entorhinal cortex receives sensory information from the association areas of the frontal lobe, parietal lobe, and temporal lobe. The information is converted into memory through the Papez circuit.

Short-term memory (episodic memory) is facilitated by unidirectional activation of synaptic connection as follows:

(i) Activation of the entorhinal cortex (parahippocampal gyrus) by input from the neocortex and limbic system.

(ii) Stimuli pass from the entorhinal cortex to the dentate gyrus via perforant path and then pass through the CA3 area.

(iii) Schaffer's collaterals from the CA3 transfer stimuli to the CA1; then CA1 efferent synapse at the subiculum.

(iv) Efferent fibers from the subiculum again project back to the entorhinal cortex.

Connections between the CA3 and dentate gyrus, and between CAl and CA3, lack feedback loop. Alveus, fimbria, and the fornix formed by the axon fibers of the CA3 pyramidal cells and neurons of the subiculum are the major hippocampal output. Fibers from the pre-commissural fornix (derived from the CA3) are connected to the lateral septal nucleus, and fibers of the post-commissural fornix (derived from the subiculum) are connected to the mammillary bodies and the hypothalamic nuclei.

\section{Rapid formation of new memory}

Long-term potentiation (LTP) is a mechanism for the rapid formation of new memory. The long-term potentiation increases synaptic efficiency following the high frequency activity of the pre-synaptic terminal. This mechanism involves Schaffer's collaterals and mossy fibers of the hippocampus. This effect lasts for many days leading to increased activity of the post-synaptic neurons. The high frequency activity is responsible for the accumulation of the calcium ions in postsynaptic neurons which triggers the LTP. Even though the original external stimulus has stopped, the impulses are transmitted frequently from the synapses of the hippocampal formation in the LTP.

\section{Spatial memory}

The hippocampus contains cells (place cells) encoding the spatial memory; these cells are responsible for recalling a place and recalling a route to reach the place. 


\section{Sommer's sector}

Large pyramidal cells of the CAl (an area known as Sommer's sector) are extremely sensitive to the oxygen lack; these cells necrose within a few minutes in compromised blood supply. In a condition leading to cerebral ischemia, the subject may lose the memory of the preceding few hours of the incident.

\section{BLOOD SUPPLY AND DRAINAGE OF HIPPOCAMPUS}

Hippocampus plays an important role in the formation of memory, and the dysfunction of the hippocampus leads to neurological disorders like Alzheimer's disease and epilepsy $(1,8,29)$. Hippocampus is supplied by the branches of the posterior cerebral artery and anterior choroidal artery $(1,8,30)$.

\section{Posterior cerebral artery}

Posterior cerebral artery is the terminal branch of the basilar artery. It joins with the posterior communicating artery to complete the circle of Willis $(1,8)$. The posterior cerebral artery can be divided into four segments, and it gives three major branches: cortical branches, postero-lateral striate branches, and posterior choroid artery $(29,30)$. The second part of the posterior cerebral artery (from the posterior communicating artery to the posterior margin of the midbrain) gives the anterior inferior temporal artery and the anterior hippocampal-parahippocampal artery which supply the entorhinal area $(1,29,30)$. The posterior parahippocampal artery arises from the posterior inferior temporal artery (branch of the posterior cerebral artery). The parietooccipital arterial trunk (the branch from the fourth part of the posterior cerebral artery) also supply the parahippocampal gyrus and hippocampus.

\section{Anterior choroid artery}

Anterior choroid artery is the branch of the internal carotid artery. It originates from the distal part of the internal carotid artery, just after the origin of the posterior communicating artery, and runs into the subarachnoid space. The segment of the anterior choroid artery up to the inferior horn of the lateral ventricle is known as cisternal segment. The anterior choroid artery gives branches to form the choroid plexus for the inferior horn of the lateral ventricle. In addition, the anterior choroid artery also supplies the optic tract, the uncus, the globus pallidus, lateral geniculate body, and the internal capsule. The perforating branches of the anterior choroid artery supplies the amygdala and the hippocampus $(1,30)$.

The anterior hippocampal arteries supply the uncus and the head of the hippocampus, while the posterior hippocampal arteries supply the body and tail of the hippocampus $(29,30)$. The anterior hippocampal arteries (branch of the anterior inferior temporal artery) enter into the uncal sulcus and supplies the head of the hippocampus. It emerges on the surface of the pyriform lobe and supplies to the adjacent entorhinal area. The posterior hippocampal arteries run in the superficial course of the hippocampal sulcus; along the terminal segment, it gives longitudinal 
large and small branches. The large branches penetrate the hippocampus and small branches supply the margo denticulatus and fimbriodentate sulcus. The longitudinal branches form a rich anastomosis along the hippocampal sulcus $(1,29,30)$.

The intrahippocampal arteries (deep branches) can be classified as large ventral, small ventral, large dorsal, and small dorsal branches. The large ventral intrahippocampal arteries supply stratum lacunosum, stratum pyramidalis, molecular layer of dentate gyrus, and CA1 and CA2 region of the cornu ammonis. The large dorsal intrahippocampal arteries supply the granular layer of the dentate gyrus, and CA3 and CA4 regions of the cornu ammonis. The small ventral intrahippocampal arteries supply the proximal part of the dentate gyrus. The small dorsal intrahippocampal arteries (also known as straight arteries) runs into the fibriodentate sulcus and supplies the adjacent areas.

\section{Venous drainage}

The deep hippocampal veins (intrahippocampal veins) are two types: sulcal intrahippocampal veins and subependymal intrahippocampal veins. The sulcus intrahippocampal veins originate from the CA1 and CA2 areas and reaches the superficial hippocampal sulcus and receive tributaries from the stratum moleculare. The subependymal intrahippocampal veins can be observed on the ventricular surface of the hippocampus. The deep hippocampal veins of CA2 and subiculum drain into the subependymal intrahippocampal veins $(1,29,30)$. Superficial hippocampal veins form two longitudinal superficial venous arcade to cover the fimbriodentate sulcus and the superficial hippocampal sulcus. The venous arcade of the fimbriodentate sulcus receives subependymal intrahippocampal veins. The venous arcade of the superficial hippocampal sulcus receives deep intrahippocampal veins $(29,30)$. Both longitudinal superficial venous arcades unite at the anterior and posterior ends. Anterior end drains into the inferior ventricular vein and posteriorly drain into medial atrial vein. Inferior ventricular vein and medial atrial vein drain into the basal vein.

The blood vessels supplying the hippocampus have small calibers and are more prone to thrombus formation. The thrombosis of the hippocampal arteries leads to damage and death of pyramidal neurons of the hippocampus which is characteristic for Alzheimer's disease.

\section{CONCLUSION}

Hippocampal formation is a crucial structure for the memory processing and emotional integration. Bilateral hippocampal damage may result in antegrade amnesia; the condition in which the brain fails to establish new long-term memories.

Conflict of Interest: The authors declare no potential conflicts of interest with respect to research, authorship and/or publication of this manuscript.

Copyright and Permission Statement: The authors confirm that the materials included in this chapter do not violate copyright laws. Where relevant, appropriate 
permissions have been obtained from the original copyright holder(s), and all original sources have been appropriately acknowledged or referenced.

\section{REFERENCES}

1. Tatu L, Vuillier F. Structure and Vascularization of the Human Hippocampus. Front Neurol Neurosci. 2014;34:18-25. https://doi.org/10.1159/000356440

2. Knierim JJ. The Hippocampus. Curr Biol. 2015;25(23):R1116-R1121. https://doi.org/10.1016/j. cub.2015.10.049

3. Ten Donkelaar HJ, Tzourio-Mazoyer N, Mai JK. Toward a Common Terminology for the Gyri and Sulci of the Human Cerebral Cortex. Front Neuroanat. 2018;12:93. https://doi.org/10.3389/ fnana.2018.00093

4. Dekeyzer S, De Kock I, Nikoubashman O, Bossche SV, Eetvelde RV, Groote JD et al. "Unforgettable" - A Pictorial Essay on Anatomy and Pathology of the Hippocampus. Insights Imaging. 2017;8(2):199-212. https://doi.org/10.1007/s13244-016-0541-2

5. Di Ieva A, Fathalla H, Cusimano MD, Tschabitscher M. The Indusium Griseum and the Longitudinal Striae of the Corpus Callosum. Cortex. 2015;62:34-40. https://doi.org/10.1016/j.cortex.2014.06.016

6. Pavlovic S, Stefanovic N, Malobabic S, Babic Z, Kostić A, Pavlovic M. Longitudinal Striae of the Human Fornix: Shape, Relations and Variations. Surg Radiol Anat. 2009;31(7):501-506. https://doi. org/10.1007/s00276-009-0471-5

7. Di Ieva A, Tschabitscher M, Rodriguez y Baena R. Lancisi's Nerves and the Seat of the Soul. Neurosurgery. 2007;60(3):563-568. https://doi.org/10.1227/01.NEU.0000249283.46514.93

8. Laplante F, Mnie-Filali O, Sullivan RM. A Neuroanatomical and Neurochemical Study of the Indusium Griseum and Anterior Hippocampal Continuation: Comparison with Dentate Gyrus. J Chem Neuroanat. 2013;50-51:39-47. https://doi.org/10.1016/j.jchemneu.2013.03.004

9. Patra DP, Tewari MK, Sahni D, Mathuriya SN. Microsurgical Anatomy of Medial Temporal Lobe in North-West Indian Population: Cadaveric Brain Dissection. Asian J Neurosurg. 2018;13(3):674-680. https://doi.org/10.4103/1793-5482.238077

10. Fogwe LA, Reddy V, Mesfin FB. Neuroanatomy, Hippocampus. In: StatPearls. Treasure Island (FL): StatPearls Publishing; February 15, 2021.

11. Parmar SK, Pruthi N, Ravindranath R, Ravindranath Y, Somanna S, Philip M. Anatomical Variations of the Temporomesial Structures in Normal Adult Brain - A Cadaveric Study. J Neurosci Rural Pract. 2018;9(3):317-325. https://doi.org/10.4103/jnrp.jnrp_73_18

12. Fu TY, Ho CR, Lin CH, Lu YT, Lin WC, Tsai MH. Hippocampal Malrotation: A Genetic Developmental Anomaly Related to Epilepsy? Brain Sci. 2021;11(4):463. Published 2021 Apr 5. https://doi.org/ 10.3390/brainscil 1040463

13. Wilczyńska K, Simonienko K, Konarzewska B, Szajda SD, Waszkiewicz N. Morphological changes of the brain in mood disorders. Zmiany morfologiczne mózguw zaburzeniach nastroju. Psychiatr Pol. 2018;52(5):797-805. https://doi.org/10.12740/PP/89553

14. Ho NF, Hooker JM, Sahay A, Holt DJ, Roffman JL. In Vivo Imaging of Adult Human Hippocampal Neurogenesis: Progress, Pitfalls and Promise. Mol Psychiatry. 2013;18(4):404-416. https://doi. org/10.1038/mp.2013.8

15. Mercer A, Thomson AM. Cornu Ammonis Regions-Antecedents of Cortical Layers? Front Neuroanat. 2017;11:83. https://doi.org/10.3389/fnana.2017.00083

16. Mazher KM, Hassan RM. Histological, Histochemical, and Immunohistochemical Studies of Hippocampus in Male New Zealand rabbits. Anat Rec (Hoboken). 2021;304(2):393-399. https://doi. org/10.1002/ar.24418

17. Lacaille JC, Schwartzkroin PA. Stratum lacunosum-moleculare interneurons of hippocampal CAI region. I. Intracellular Response Characteristics, Synaptic responses, and Morphology. J Neurosci. 1988;8(4):1400-1410. https://doi.org/10.1523/JNEUROSCI.08-04-01400.1988 
18. Carlson ML, Toueg TN, Khalighi MM, Castillo J, Shen B, Azevedo E. et al. Hippocampal subfield imaging and fractional anisotropy show parallel changes in Alzheimer's disease tau progression using simultaneous tau-PET/MRI at 3T. Alzheimers Dement (Amst). 2021;13(1):e12218. https://doi. org/10.1002/dad2.12218

19. Li MM, Fan JT, Cheng SG, Yang LF, Yang L, Wang LF. et al. Enhanced Hippocampus-Nidopallium Caudolaterale Connectivity during Route Formation in Goal-Directed Spatial Learning of Pigeons. Animals (Basel). 2021;11(7):2003. https://doi.org/10.3390/anil1072003

20. Vorkapic C, Leal S, Alves H, Douglas M, Britto A, Dantas EHM. Born to move: a review on the impact of physical exercise on brain health and the evidence from human controlled trials. Arq Neuropsiquiatr. 2021;79(6):536-550. https://doi.org/10.1590/0004-282X-ANP-2020-0166

21. Yu W, Wu X, Chen Y, Liang Z, Jiang J, Misrani A, et al. Pelvic Pain Alters Functional Connectivity Between Anterior Cingulate Cortex and Hippocampus in Both Humans and a Rat Model. Front Syst Neurosci. 2021;15:642349. https://doi.org/10.3389/fnsys.2021.642349

22. Martens M, Filippini N, Masaki C, Godlewska BR. Functional Connectivity between Task-Positive Networks and the Left Precuneus as a Biomarker of Response to Lamotrigine in Bipolar Depression: A Pilot Study. Pharmaceuticals (Basel). 2021;14(6):534. https://doi.org/10.3390/ph14060534

23. Ballotta D, Talami F, Pizza F, Vaudeno AE, Benuzzi F, Plazzi G et al. Hypothalamus and Amygdala Functional Connectivity at Rest in Narcolepsy Type 1 [published online ahead of print, $2021 \mathrm{Jul} 4$ ]. Neuroimage Clin. 2021;31:102748. https://doi.org/10.1016/j.nicl.2021.102748

24. Cascella M, Al Khalili Y. Short Term Memory Impairment. 2021 Jul 21. In: StatPearls [Internet]. Treasure Island (FL): StatPearls Publishing; 2021 Jan-. PMID: 31424720.

25. Vann SD, Nelson AJ. The Mammillary Bodies and Memory: more than a Hippocampal Relay. Prog Brain Res. 2015;219:163-185. https://doi.org/10.1016/bs.pbr.2015.03.006

26. Taghipour M, Derakhshan N. Joy of Learning: Mammilotegmental Tract Connecting 2 Circuits of Memory and Pleasure in Brain. World Neurosurg. 2018;118:387-388. https://doi.org/10.1016/j. wneu.2018.06.069

27. Dillingham CM, Frizzati A, Nelson AJ, Vann SD. How do Mammillary Body inputs Contribute to Anterior Thalamic Function? Neurosci Biobehav Rev. 2015;54:108-119. https://doi.org/10.1016/j. neubiorev.2014.07.025

28. Dillingham CM, Milczarek MM, Perry JC, Vann SD. Time to Put the Mammillothalamic Pathway into Context. Neurosci Biobehav Rev. 2021;121:60-74. https://doi.org/10.1016/j.neubiorev.2020.11.031

29. Rusinek H, Brys M, Glodzik L, Switalski R, Tsui WH, Haas F et al. Hippocampal blood flow in normal aging measured with arterial spin labeling at 3T. Magn Reson Med. 2011;65(1):128-137. https://doi. org/10.1002/mrm.22611

30. Isolan GR, Stefani MA, Schneider FL, Claudino HA, Yu YH, Choi GG, et al. Hippocampal vascularization: Proposal for a new classification. Surg Neurol Int. 2020;11:378. Published 2020 Nov 6. https:// doi.org/10.25259/SNI_708_2020. 